\title{
Probabilistic-Driven Oriented Speckle Reducing Anisotropic Diffusion with Application to Cardiac Ultrasonic Images
}

\author{
G. Vegas-Sanchez-Ferrero ${ }^{1, \star}$, S. Aja-Fernandez ${ }^{1}$, M. Martin-Fernandez ${ }^{1}$, \\ A.F. Frangi ${ }^{3}$, and C. Palencia ${ }^{2}$ \\ 1 Laboratorio de Procesado de Imagen, Universidad de Valladolid, Spain \\ 2 Dep. Matematica Aplicada, Universidad de Valladolid, Spain \\ 3 CISTIB, Universidad Pompeu Fabra, Spain
}

\begin{abstract}
A novel anisotropic diffusion filter is proposed in this work with application to cardiac ultrasonic images. It includes probabilistic models which describe the probability density function (PDF) of tissues and adapts the diffusion tensor to the image iteratively. For this purpose, a preliminary study is performed in order to select the probability models that best fit the stastitical behavior of each tissue class in cardiac ultrasonic images. Then, the parameters of the diffusion tensor are defined taking into account the statistical properties of the image at each voxel. When the structure tensor of the probability of belonging to each tissue is included in the diffusion tensor definition, a better boundaries estimates can be obtained instead of calculating directly the boundaries from the image. This is the main contribution of this work. Additionally, the proposed method follows the statistical properties of the image in each iteration. This is considered as a second contribution since state-ofthe-art methods suppose that noise or statistical properties of the image do not change during the filter process.
\end{abstract}

Keywords: Anisotropic diffusion, probability models, diffusion tensor, structure tensor, cardiac ultrasonic images.

\section{Introduction}

Ultrasound is a non-invasive imaging modality and a low-cost way to help diagnosing, which is widespread for many medical applications. However, ultrasonic (US) images are characterized by the presence of speckle, which is a granular pattern that degrades resolution and adds spatial noise to the image 11. Therefore, many speckle removal filters have been proposed in the literature 234.

Speckle in ultrasound images can be seen as a random process whose statistical features depend on the tissue class. The existence of a deterministic component

\footnotetext{
* Thanks to Gerencia Regional de Salud, Consejeria de Sanidad y Junta de Castilla y Leon (GRS 292/A/08, SAN126/VA32/09, SAN126/VA33/09, VA027/A07) and Ministerio de Ciencia e Innovacion (MTM2007-63257) for funding.
}

T. Jiang et al. (Eds.): MICCAI 2010, Part I, LNCS 6361, pp. 518-525, 2010.

(C) Springer-Verlag Berlin Heidelberg 2010 
due to specular reflections of the echo-pulse depends on the number of obstacles of the tissue (scatters) into the resolution cell and their size in comparison with the wavelength of ultrasound signal. Thus, many of the speckle removal filters are based on local statistics.

Among those filters, we focus on anisotropic diffusion based filters since the proposed filter can be considered as a probabilistic extension of them. One of the most popular approaches is Speckle Reducing Anisotropic Diffusion (SRAD) 2, which extends the well known Perona Malik's anisotropic diffusion avoiding to threshold the norm of the gradient. Instead of that threshold, an estimate of the coefficient of variation of noise is used. Results and stability depends on a good estimation of the local statistics as it was demonstrated in [3].

In the case of Oriented Speckle Reducing Anisotropic Diffusion (OSRAD) 4, no logarithmic compression is supposed and the speckle statistics are assumed to be modeled by a Rician distribution. In the case of high SNR, speckle statistics are approximated by a Gaussian distribution. This is a reasonable assumption when resolution cells have a large number of scatters and there exists a deterministic component, which is the case of the myocardial tissue for the raw signal obtained by the transducer. However, besides logarithmic compression, an interpolation process is performed to reconstruct the image and, therefore, the probabilistic model is affected. Additionally, the diffusion schemes are iterative, so the Rician distribution supposition does not hold in each iteration. So, because of logarithmic compression and interpolation, the statistics of speckle in reconstructed images do not follow the original distributions of the raw signal. Hence, many recent works study different probability models in order to obtain a suitable model to describe the statistical behavior of the speckle [15]6].

In this work, we propose a novel anisotropic diffusion filter with application to cardiac ultrasonic images, which includes the probability models that describe PDFs of different tissue classes and adapt iteratively to the filtered image. Probability models for each tissue class were selected from a training database using real-life cases for cardiac ultrasonic images. A mixture PDF model is adopted for fitting probabilistic models of tissue classes to the histogram of the whole image. With this methodology, a Bayesian scheme can be applied to estimate the probability of belonging to each of the tissue classes for every image voxel.

When the structure tensor of the probability of belonging to each tissue is combined in the definition of the diffusion tensor, better boundaries estimates can be obtained instead of directly calculating them from the image. The structure tensor of the probability of belonging to each tissue allows defining a diffusion tensor, which takes into consideration the boundaries between different tissue classes for filtering purposes. This is the main contribution of this work and, to the best of our knowledge, no similar approach has been considered in the literature for this purpose.

The paper is organized as follows. In section 2, the stasticial study is explained and the distributions are selected. Section 3 describes the filtering method. Section 4 presents some experiments and results on synthetic and real images. In section 5, we conclude analyzing the results. 


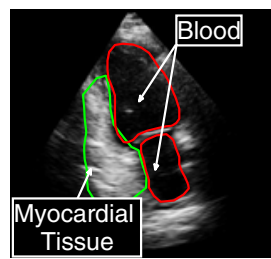

(a)

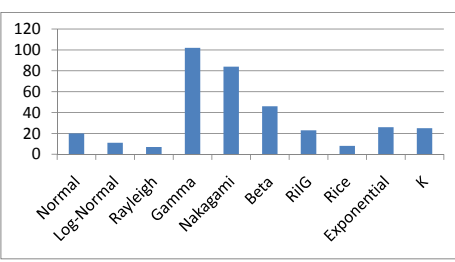

(b)

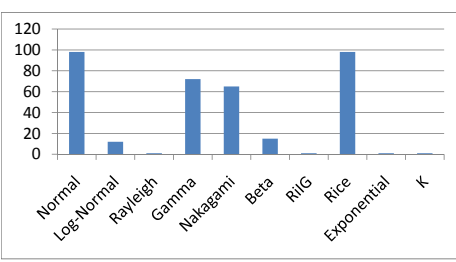

(c)

Fig. 1. (a) Example of the regions for blood and myocardial tissue. (b) Blood. (c) Myocardial Tissue. For blood tissue class, the Gamma distribution performs best. For myocardial tissue, the best performance is achieved for Rice or normal distributions.

\section{Probabilistic Estimation of Tissue Classes in US Images}

In this section, the goodness-of-fit of several probabilistic distributions is presented. The study is extended to a representative set of distributions that were proposed in the literature: Gamma [15], Log-Normal [5], Rayleigh 1156], Normal [5], Nakagami [16], Beta, Rician Inverse Gaussian (RiIG) 6], Rice [7], Exponential and K [1]. A set of 120 two-dimensional images of size $1024 \times 768$ and 8 bits per pixel were obtained from a clinical machine Philips iE33 ultrasonographic system 11 with the software PMS5.1 Ultrasound iE33 4.0.1.357 scanned from human subjects.

The methodology used to carry out the study is the same used in [1.5] where a $\chi^{2}$ goodness-to-fit test is performed. We used a significance value $\alpha=0.05$ for the test. In order to avoid spatial correlation, the image is subsampled by a factor of 6 . Two different tissue classes were considered: blood and myocardium.

In Fig. 1, an example of the analyzed tissues is presented as well as the results of passing the goodness-of-fit test for blood an myocardial tissue. In Fig. 1.b we can see the better performance of the gamma distribution in the case of blood, this result holds with that one obtained in [5]. In the case of the myocardial tissue (Fig. 1.c), Rice distribution and Normal distributions are the ones with the best fit. This result is coherent to the interpretation of quasi periodic scatterers, which gives rise to a Rician model of speckle [7] and confirms the hypothesis of the OSRAD filter [4. Due to the simplicity of estimating Normal parameters for estimating mixtures of PDFs, we chose the Normal distribution as a good candidate for this tissue class.

\section{Probabilistic Directional SRAD}

When the histogram of the ultrasonic image is considered as the contribution of different kinds of tissues which follow different PDFs, the mixture of PDFs arises as a natural way to fit the histogram. This way, when parametric distributions are considered, the parameters of each independent variable have to be calculated.

\footnotetext{
${ }^{1}$ Philips Healthcare, Andover, MA, USA.
} 


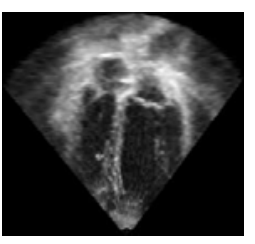

(a)

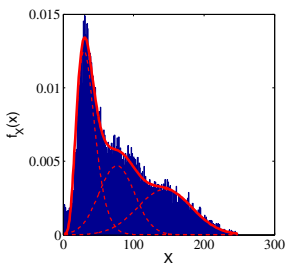

(b)

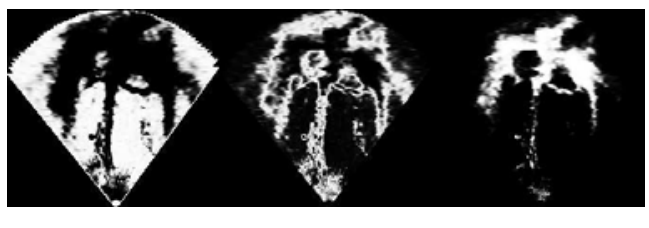

(c)

Fig. 2. (a) Clinical ultrasound image. (b) Finite Mixture Model. (c) Tissue class probability map for each voxel of the image. The good fit of the mixture model of the image (a) becomes clear as well as the structure of the ownership to each class where the boundaries of the left and right ventricles are clearly defined.

The most widespread method for this purpose is the Expectation-Maximization (EM) algorithm, which allows estimating the parameters of the mixture. We decided to adopt the unsupervised learning of mixture models method [8], since it is capable of selecting the number of mixture components from a maximum $\left(k_{\max }\right)$ and it simultaneously integrates model selection and estimation.

Let $X$ be a random variable (and $x$ a sample) that follows a finite mixture PDF of $K$ components where $K \in\left[1, k_{\max }\right]$. Its PDF can be written as follows:

$$
f_{X}(x)=\sum_{k=1}^{K} a_{k} f_{X_{k}}\left(x \mid \Theta_{k}\right)
$$

where $a_{k}>0$ and $\sum_{k=1}^{K} a_{k}=1$ are the probabilities of belonging to each class of the mixture, and $\Theta_{k}$ are the parameters of the class $\mathcal{C}_{k}$, which follow a Gaussian or Gamma distribution. The probability of belonging to each class for each voxel (i.e. each sample $x$ ) is the following:

$$
P\left(x \in \mathcal{C}_{k} \mid X=x, \Theta_{k}\right)=\frac{P\left(X=x \mid x \in \mathcal{C}_{k}, \Theta_{k}\right) P\left(x \in \mathcal{C}_{k} \mid \Theta_{k}\right)}{\sum_{i=1}^{K} P\left(X=x \mid x \in \mathcal{C}_{i}, \Theta_{i}\right) P\left(x \in \mathcal{C}_{i} \mid \Theta_{i}\right)}
$$

This expresion allows us to calculate a set of images that indicate the probability of belonging to each class for every voxel and can be seen as the partial volume contribution of each class. Fig 2 shows an ultrasound image, its finite mixture PDF, and the probability ownership to each class. In Fig 2 b a good fit of the mixture model of the image in Fig. 2, a becomes clear as well as the structure the ownership to each class (Fig. 2.c).

The main advantage of this set of images is that a structure tensor can be derived from each probability image, which is able to extract the directions of minimal probability change even when low gradients are presented. This way, an anisotropic filter can be defined that takes the advantage of these directions in the probability map for each tissue class. We will refer to it as POSRAD (Probabilistic Oriented Speckle Reducing Anisotropic Diffusion). This filter makes use 
of the extension of the scalar component of the diffusion equation to a diffusion matrix which takes the advantage of the local tissue orientation as it was proposed in 49. However, in our case we calculate the local orientation of the probability density function for each tissue class and combine them in the following way.

As in [9], we calculate the structure tensor of the probability density function for each tissue class as:

$$
T_{k}=G_{\sigma} *\left(\nabla_{\sigma} P\left(x \in \mathcal{C}_{k} \mid X=x, \Theta_{k}\right) \cdot \nabla_{\sigma} P\left(x \in \mathcal{C}_{k} \mid X=x, \Theta_{k}\right)^{T}\right)
$$

where $G_{\sigma}$ is a Gaussian kernel of standard deviation $\sigma$, and $\nabla_{\sigma} P\left(x \in \mathcal{C}_{k} \mid X=\right.$ $\left.x, \Theta_{k}\right)$ is the gradient of the probability density function for each tissue class filtered with a Gaussian kernel of standard deviation $\sigma$. Finally, let $\lambda_{1}^{k} \geq \lambda_{2}^{k} \geq$ $\lambda_{3}^{k}$ be the eigenvalues and $\left(\mathbf{v}_{1}^{k}, \mathbf{v}_{2}^{k}, \mathbf{v}_{3}^{k}\right)$ their respective eigenvectors. The local orientation of the maximal variation of probability of the class $\mathcal{C}_{k}$ is given by $\mathbf{v}_{1}^{k}$, and the local orientation of the minimal variation is given by $\mathbf{v}_{3}^{k}$.

Let consider the following diffusion equation:

$$
\left\{\begin{aligned}
u(x, 0) & =u_{0} \\
\frac{\partial u}{\partial t} & =\operatorname{div}(D \nabla u)
\end{aligned}\right.
$$

where the matrix $D$ is expressed in a diagonal form with the eigenvectors $\left(\mathbf{v}_{1}, \mathbf{v}_{2}\right.$, $\mathbf{v}_{3}$ ) and eigenvalues $\lambda_{1}, \lambda_{2}, \lambda_{3}$. Since we have $K$ structure tensors (each tissue class with probability density function), we choose the eigenbase of the structure tensor with maximal $\lambda_{1}^{k}: \hat{k}=\arg \max _{k}\left(\lambda_{1}^{k}\right)$. This base gives the orientation of the maximal variation of probability among all the classes. The interpretation of this choice is that we choose as boundary the one with the maximal gradient of the probability density function over all tissue classes. This way, the most probable boundary is preserved in the filtering process. In the basis of $T_{\hat{k}}$, namely $\left(\mathbf{e}_{1}, \mathbf{e}_{2}, \mathbf{e}_{3}\right)$, the diffusion matrix $D$ is defined as:

$$
D=E\left(\begin{array}{ccc}
\lambda_{1} & 0 & 0 \\
0 & \lambda_{2} & 0 \\
0 & 0 & \lambda_{3}
\end{array}\right) E^{T} \quad \begin{aligned}
& \lambda_{1}=1-\left\|\nabla_{\mathbf{e}_{1}, \sigma} P\left(x \in \mathcal{C}_{\hat{k}} \mid X=x, \Theta_{\hat{k}}\right)\right\|_{2} \\
& \lambda_{2}=1-\left\|\nabla_{\mathbf{e}_{2}, \sigma} P\left(x \in \mathcal{C}_{\hat{k}} \mid X=x, \Theta_{\hat{k}}\right)\right\|_{2} \\
& \lambda_{3}=1
\end{aligned}
$$

where $\|\cdot\|_{2}$ is the 2-norm, $\nabla_{\mathbf{e}_{i}, \sigma}$ is the directional derivative in the direction $\mathbf{e}_{i}$ filtered with a Gaussian kernel with a standard deviation $\sigma$, and $E$ is the matrix whose columns are the eigenvectors $\left(\mathbf{e}_{1}, \mathbf{e}_{2}, \mathbf{e}_{3}\right)$. Note that this definition performs a diffusion filtering in the direction of the minimal variation of probability $\left(\mathbf{e}_{3}\right)$ while preserves the maximal variation of probability since $\| \nabla_{\mathbf{e}_{1}, \sigma} P(x \in$ $\left.\mathcal{C}_{\hat{k}} \mid X=x, \Theta_{\hat{k}}\right) \|_{2}$ will have a value closed to 1 . Note that the discrete approximations of $\left\|\nabla_{\mathbf{e}_{1}, \sigma} P\left(x \in \mathcal{C}_{\hat{k}} \mid X=x, \Theta_{\hat{k}}\right)\right\|_{2}$ and $\left\|\nabla_{\mathbf{e}_{2}, \sigma} P\left(x \in \mathcal{C}_{\hat{k}} \mid X=x, \Theta_{\hat{k}}\right)\right\|_{2}$ are bounded in $[0,1]$, thus $\lambda_{1}, \lambda_{2} \in[0,1]$.

The main advantage of this definition is that, in homogeneous areas $D$ becomes isotropic and in the presence of boundaries it becomes more anisotropic and with a main orientation aligned along the boundaries thus preserving the main contours. Additionally, the evolution of the probabilistic models can be followed through the iterative process since the finite mixture model is re-estimated 
in each iteration. This is an important advantage of the presented methodology over other state-of-the-art filters, which assume that the stastitics of the image/noise do not change during the filtering process. Since no assumptions can be made about the changes of the statistics of the image, a finite mixture of Gaussians is adopted during the filtering process except for the first iteration.

\section{Experiments and Results}

In this section we present some experiments with synthetic and real ultrasound images in order to compare our technique to other state-of-the-art algorithms. First we consider the 2D synthetic image presented in 4 which is publicly available 2 and allows us to compare the proposed method to other algorithms. This image is presented in Fig 3. a which has 6 regions of constant intensity. The image is corrupted with a Gaussian multiplicative noise with $\sigma_{n}=0.5$ (Fig. 3.b).In Fig. 3. c the filtered image with the proposed method is presented. The parameters of the filter were: 200 iterations, time step $0.5, \sigma=1$ and $k_{\max }=6$.

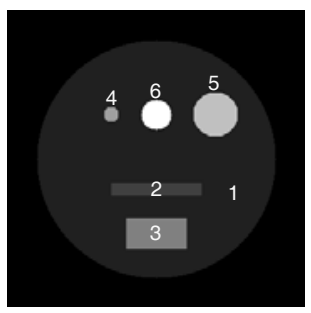

(a)

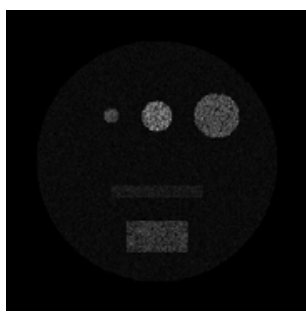

(b)

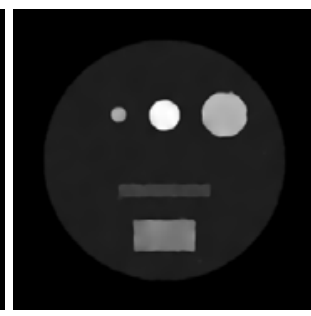

(c)

Fig. 3. (a) Original synthetic image of [4]. (b) Noisy image with multiplicative noise with $\sigma=0.5$. (c) Filtered image with the proposed filter.

Table 1. Results on filtering synthetic $2 \mathrm{D}$ image with $\sigma_{n}=0.5$, presented as Mean \pm Standard Deviation of the intensity in each region (R1 - R6) of the Fig. 3,a

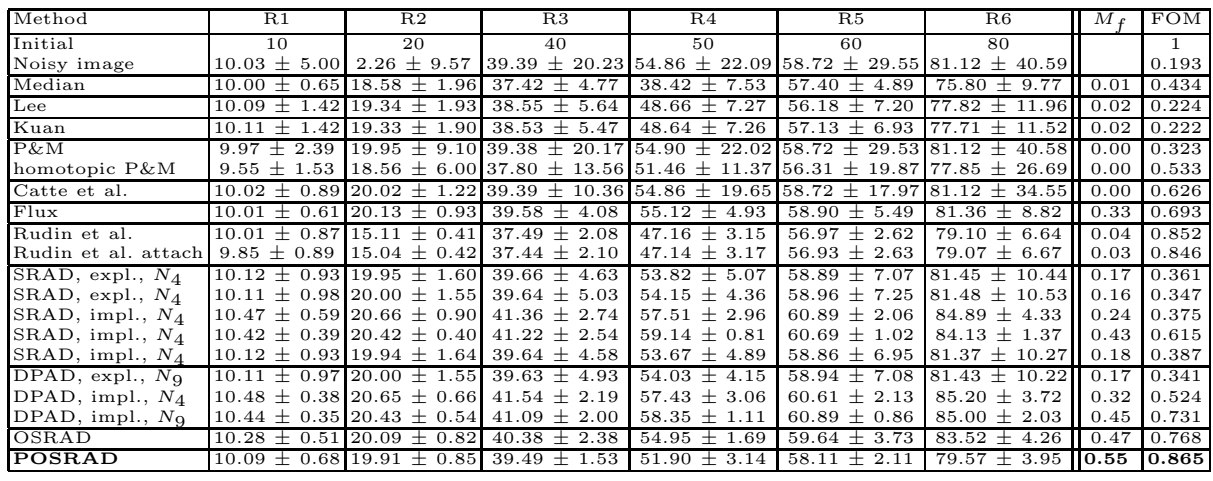

${ }^{2}$ http://serdis.dis.ulpgc.es/ krissian/HomePage/Demos/OSRAD/OSRAD.html 

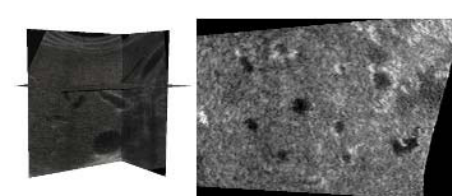

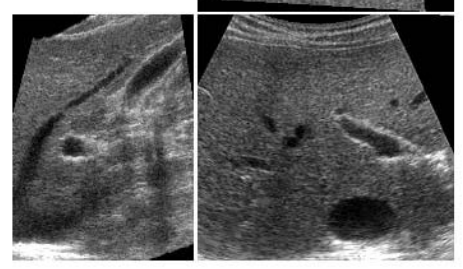

(a)

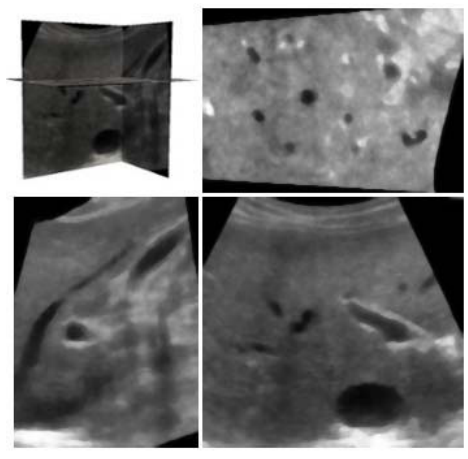

(b)

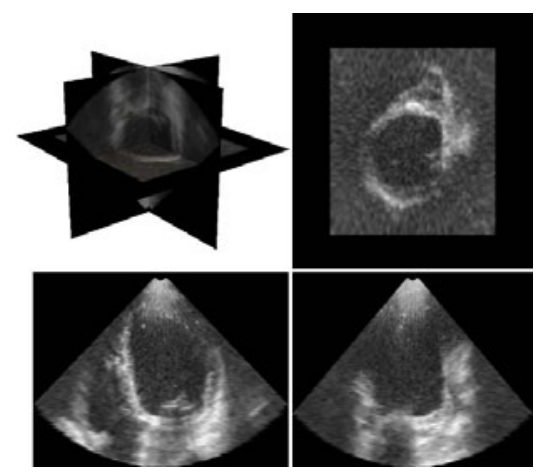

(c)

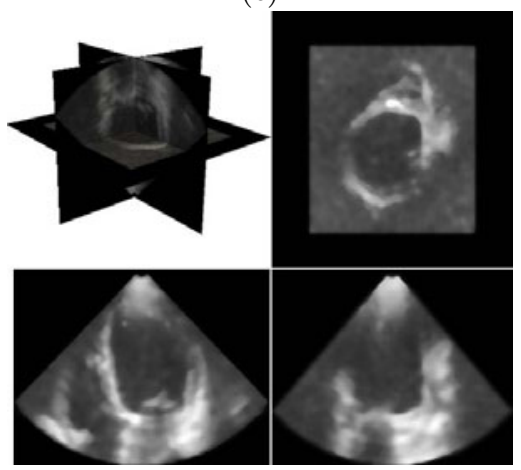

(d)

Fig. 4. (a) Noisy 3D ultrasound of a liver. (b) Filtered 3D ultrasound of a liver. (c) Noisy 3D ultrasound of a Heart. (d) Filtered 3D ultrasound of a Heart. The proposed method achieve a good edge preservation with an efficient noise removal in both cases.

No suppositions were made concerning the probability distributions of the tissue classes, so a Gaussian mixture model was used.

Since these parameters are the same used by OSRAD, a direct comparison with the results in 4 can be established. We use the same quality measures for validation: $M_{f}$ and figure of merit $(\mathrm{FOM}) \sqrt{3}$, see [4] for more details. Both values are comprised between 0 and 1 . The highest the value, the better the filter. Results presented in Table 1 show that the best FOM and $M_{f}$ is achieved by our method. These results show that a better performance can be achieved when variations of the probability for each class is taken into consideration since the diffusion tensor becomes more anisotropic near the most probable edges while homogeneous regions are filtered in an isotropic way.

We now consider a 3D ultrasound image of a liver generated using the Stradwin v3.8 software (Cambridge University, Cambridge, UK), which has been used

${ }^{3}$ The quality measure $M_{f}$ was recalculated since it measures the quality of the filter compared to the others filters. 
commonly in the literature [4]. These data were acquired by a freehand system and are available on Cambridge University 4 . The image is a volume with $201 \times 193 \times 142$ voxels with an isotropic resolution of $0.5 \times 0.5 \times 0.5 \mathrm{~mm}^{3}$. Both, the original and the filtered image are presented in Fig 4 (a-b). Visual inspection shows that a good edge preservation is obtained with an efficient noise removal. The parameters for this experiment were: 200 iterations, time step $0.5, \sigma=1$ and $k_{\max }=6$ with a Gaussian mixture model.

As a final experiment, we consider a real 3D ultrasonic image of the heart obtained with the same clinical machine of section 2 . In this experiment we use the finite mixture model of Gaussian and Gamma distributions, 100 iterations, time step $0.5, \sigma=1$ and $k_{\max }=4$. Results are presented in Fig. 4. (c-d) were a good edge preservation is observed. An efficient noise removal is achieved in the left ventricle when compared to the noisy image.

\section{Conclusion}

In this work we have presented a new diffusion filter that takes into account the statistical properties of the image. This methodology offers an advantage over the follow-up of statistical properties of the image in each iteration while preserving and enhancing the structures. Experiments on synthetic and real images show that the proposed method preserves edges of the structures better than others and performs a good restoration.

\section{References}

1. Nillesen, M.M., Lopata, R.G., Gerrits, I.H., Kapusta, L., Thijssen, J.M., de Korte, C.L.: Modeling envelope statistics of blood and myocardium for segmentation of echocardiographic images. Ultrasound Med. Biol. 34(4), 674-680 (2008)

2. Yu, Y., Acton, S.: Speckle reducing anisotropic diffusion. IEEE Trans. Img. Proc. 11(11), 1260-1270 (2002)

3. Aja-Fernandez, S., Alberola-Lopez, C.: On the estimation of the coefficient of variation for anisotropic diffusion speckle filtering. IEEE Trans. Img. Proc. 15(9), 2694 2701 (2006)

4. Krissian, K., Westin, C.F., Kikinis, R., Vosburgh, K.: Oriented speckle reducing anisotropic diffusion. IEEE Trans. Img. Proc. 16(5), 1412-1424 (2007)

5. Tao, Z., Tagare, H.D., Beaty, J.D.: Evaluation of four probability distribution models for speckle in clinical cardiac ultrasound images. IEEE Trans. Med. Imag. 25(11), 1483-1491 (2006)

6. Eltoft, T.: Modeling the amplitude statistics of ultrasonic images. IEEE Trans. Med. Imag. 25(2), 229-240 (2006)

7. Wagner, R., Smith, S., Sandrik, J., Lopez, H.: Statistics of speckle in ultrasound B-scans. IEEE Trans. Sonics Ultrason. 30(3), 156-163 (1983)

8. Figueiredo, M., Jain, A.: Unsupervised learning of finite mixture models. IEEE Transactions on Pattern Analysis and Machine Intelligence 24, 381-396 (2002)

9. Krissian, K., Aja-Fernández, S.: Noise-driven anisotropic diffusion filtering of MRI. IEEE Trans. Img. Proc. 18(10), 2265-2274 (2009)

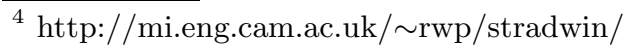

